\title{
The prognosis and effects of local treatment strategies for orbital embryonal rhabdomyosarcoma: a population-based study
}

This article was published in the following Dove Press journal:

Cancer Management and Research

\author{
Li-Ying Tang' \\ Mou-Xin Zhang ${ }^{2}$ \\ $\mathrm{Di}-\mathrm{Han} \mathrm{Lu}^{3}$ \\ Yong-Xiong Chen' \\ Zu-Guo Liu',2 \\ San-Gang $\mathrm{Wu}^{4}$
}

'Eye Institute of Xiamen University, Fujian Provincial Key Laboratory of Ophthalmology and Visual Science, Medical College, Xiamen University, Xiamen 361005, People's Republic of China; ${ }^{2}$ Xiamen University Affiliated Xiamen Eye Center, Xiamen 36I00I, People's Republic of China; ${ }^{3}$ Department of Anesthesiology, The First Affiliated Hospital, Sun Yat-Sen University, Guangzhou 510080 , People's Republic of China; ${ }^{4}$ Department of Radiation Oncology, Xiamen Cancer Hospital, the First Affiliated Hospital of Xiamen University, Xiamen 361003, People's Republic of China

Correspondence: Zu-Guo Liu Eye Institute of Xiamen University, Fujian Provincial Key Laboratory of Ophthalmology and Visual Science, Medical College, Xiamen University, Xiang'an South Road, Xiang'an District, Xiamen 36I I02, People's Republic of China

Tel +865922183761

Fax +865922183761

Email zuguoliu@xmu.edu.cn

San-Gang Wu

Department of Radiation Oncology,

Xiamen Cancer Hospital, the First

Affiliated Hospital of Xiamen University,

55 Zhenhai Road, Xiamen 361003,

People's Republic of China

Tel +86 5922139531

Fax +86592 23I 730I

Email unowu12345@hotmail.com
Introduction: Orbital embryonal rhabdomyosarcoma is a rare childhood malignancy with a good prognosis, but the optimal treatment remains unclear. Using a population-based cancer registry, we assessed the prognoses and survival outcomes of patients with orbital embryonal rhabdomyosarcoma according to the local treatment strategy.

Patients and methods: Patients diagnosed with orbital embryonal rhabdomyosarcoma between 1988 and 2012 as part of the Surveillance Epidemiology and End Results program were included. Univariate and multivariate Cox regression analyses were performed to determine the prognostic factors associated with cause-specific survival (CSS) and overall survival (OS). Results: In total, 102 patients were included; their median age was 6 years, $78.4 \%$ were white, and $56.9 \%$ were male. The median tumor size was $30 \mathrm{~mm}$. Of 20 patients with an available histologic grade, the tumors of $90 \%$ were poorly differentiated/undifferentiated. Of 92 patients with available surgical and radiotherapy (RT) statuses, 50 (54.3\%), $36(39.1 \%)$, and $6(6.5 \%)$ received surgery and RT, primary RT, and primary surgery, respectively. Ninety-five patients (93.1\%) received chemotherapy. The 5- and 10-year CSSs of the entire cohort were $94.3 \%$ and $92.2 \%$, respectively. The 5- and 10-year OSs were $93.3 \%$ and $91.3 \%$, respectively. In 95 patients who were followed up for at least 12 months, there were no significant prognostic factors related to CSS and OS. Furthermore, the local treatment strategy did not significantly affect CSS $(P=0.29)$ or OS $(P=0.468)$.

Conclusion: There is no local treatment of choice for orbital embryonal rhabdomyosarcoma in terms of survival. However, RT is a reasonable alternative treatment to surgery.

Keywords: orbital embryonal rhabdomyosarcoma, survival, radiotherapy, surgery, SEER

\section{Introduction}

Orbital embryonal rhabdomyosarcoma is a highly malignant tumor composed of differentiated striated muscle cells. ${ }^{1}$ It usually occurs in children aged $<10$ years. ${ }^{1}$ Orbital rhabdomyosarcoma has 3 histologic types: embryonal, alveolar, and pleomorphic. The tumor may appear anywhere in the orbit, but is especially common in the retrobulbar region. Embryonal rhabdomyosarcoma is the most common type of orbital rhabdomyosarcoma in children. ${ }^{2}$ In the United States, it is estimated that approximately 350 new cases of rhabdomyosarcoma, including 35 of orbital embryonal rhabdomyosarcoma, are diagnosed each year. ${ }^{3}$

In the 1960s, the overall survival (OS) of orbital rhabdomyosarcoma that received orbital exenteration was $25 \%-30 \%$. With the development of multidisciplinary treatment involving surgery, radiotherapy (RT), and chemotherapy, the OS has improved to about $90 \% .^{4-10}$ Reportedly, the 5-year OS of patients with orbital rhabdomyosarcoma 
who receive high-dose chemotherapy is $58.4 \%{ }^{6}$ Recently, a therapeutic regimen for orbital embryonal rhabdomyosarcoma was designed using the clinical grouping system of the North American Intergroup Rhabdomyosarcoma Study (IRS) and the tumor-nodes-metastasis (TNM) classification. Its comprehensive, optimal treatment strategies include surgery, RT, systemic chemotherapy, and biotherapy. ${ }^{3}$ However, the optimal local treatment for orbital embryonal rhabdomyosarcoma remains unclear. The use of RT in most patients as part of the initial management in the North American approach aims to minimize disease recurrence, whereas the European approach attempts to prevent radiation-induced effects by avoiding the use of upfront RT. ${ }^{11}$ In this study, using Surveillance Epidemiology and End Results (SEER) database, ${ }^{12}$ we investigated the prognoses and survival outcomes of patients with orbital embryonal rhabdomyosarcoma according to the local treatment strategy.

\section{Patients and methods}

We retrospectively collected data from the National Cancer Institute's SEER database, ${ }^{12}$ which is a collection of deidentified data from 18 cancer registries in the United States covering approximately $28 \%$ of the population. Patients who received a pathologic diagnosis of orbital embryonal rhabdomyosarcoma between 1988 and 2012 were included. The diagnosis was based on the primary tumor site using the third edition of the International Classification of Diseases for Oncology. We obtained permission to access the SEER database for research only (reference number: 10269-Nov2015). This study did not require patient consent because the data are deidentified, and this study was approved by the ethics committee of the First Affiliated Hospital of Xiamen University, Xiamen, Fujian, People's Republic of China.

The following demographic and clinicopathologic variables were collected: age; year of diagnosis; race; sex; histologic grade; tumor size; and SEER stage. Local treatment strategies, including primary surgery, primary RT, and surgery plus RT, were also identified.

Univariate and multivariate Cox proportional hazards regression analyses were performed to determine the prognostic factors associated with cause-specific survival (CSS) and OS. Survival curves were plotted using the Kaplan-Meier method and compared using the log-rank test. The statistical data were analyzed using SPSS version 21.0 (IBM Corporation, Armonk, NY, USA). A $P$-value of $<0.05$ was considered statistically significant.

\section{Results \\ Clinicopathologic characteristics}

In total, 102 patients with orbital embryonal rhabdomyosarcoma were included in this study. Their demographic and clinicopathologic factors are shown in Table 1. The median age of the patients was 6 years (range: $1-27$ years); $78.4 \%$ of them were white, and $56.9 \%$ were male. The median tumor size was $30 \mathrm{~mm}$ (range: $1-135 \mathrm{~mm}$ ). Of the 98 patients with an available SEER stage, 68 (66.7\%), 23 (22.5\%), and 7 $(6.9 \%)$ were at the localized, regional, and distant stages, respectively. Moreover, of the 20 patients with an available histologic grade, the tumors of $10 \%, 60 \%$, and $30 \%$ were moderately differentiated, poorly differentiated, and undifferentiated, respectively. Tumor stage was available for 45 patients: $77.8 \%$ had $\mathrm{T} 2$-stage disease. In addition, nodal stage was available for 47 patients, none of whom had nodal metastasis.

\section{Treatment}

Of the 100 and 99 patients with an available surgical or RT status, respectively, 56 (56\%) and $86(86.9 \%)$ underwent surgical and RT treatment, respectively. Of 92 patients for whom both surgical and RT statuses were available, 50 (54.3\%) received surgery and RT, 36 (39.1\%) underwent primary RT, and only $6(6.5 \%)$ received primary surgery. There were no significant differences in demographic and clinicopathologic factors between the 3 local treatment groups (Table 2). A total of 95 patients (93.1\%) received chemotherapy.

\section{Survival outcomes}

The median follow-up period was 99 months (range: 0-311 months). Nine patients died, including 8 patients as a result of malignant tumors and 1 patient as a result of heart disease. In 7 patients at the distant stage of orbital embryonal rhabdomyosarcoma, 1 patient died from heart disease, 1 patient died as a result of malignant tumors, and 5 patients are still alive (range: 52-284 months). The 5- and 10-year CSSs of the entire cohort were $94.3 \%$ and $92.2 \%$, respectively. The 5- and 10 -year OSs were $93.3 \%$ and $91.3 \%$, respectively (Figure 1A and B).

\section{Prognostic factors}

We analyzed the prognostic factors of 95 patients who were followed up for at least 12 months. The factors included in our prognostic analysis were age, race, sex, SEER stage, local treatment, and primary tumor size. In the univariate analysis, there were no significant prognostic factors related to CSS 
Table I Summary of the demographic and clinicopathologic characteristics of 102 patients

\begin{tabular}{|c|c|}
\hline Characteristics & n (\%) \\
\hline \multicolumn{2}{|l|}{ Age (years) } \\
\hline$\leq 6$ & $47(46.1)$ \\
\hline$>6$ & $55(53.9)$ \\
\hline \multicolumn{2}{|l|}{ Years of study } \\
\hline $1988-1992$ & $13(12.8)$ \\
\hline $1993-1997$ & $14(13.7)$ \\
\hline 1998-2002 & $21(20.6)$ \\
\hline $2003-2007$ & $25(24.5)$ \\
\hline $2008-2012$ & $29(28.4)$ \\
\hline \multicolumn{2}{|l|}{ Race } \\
\hline White & $80(78.4)$ \\
\hline Black & $13(12.8)$ \\
\hline Others & $9(8.8)$ \\
\hline \multicolumn{2}{|l|}{ Sex } \\
\hline Male & $58(56.9)$ \\
\hline Female & $44(43.1)$ \\
\hline \multicolumn{2}{|l|}{ Grade $(n=20)$} \\
\hline Well-differentiated & $0(0)$ \\
\hline Moderately differentiated & $2(10)$ \\
\hline Poorly differentiated & $12(60)$ \\
\hline Undifferentiated & $6(30)$ \\
\hline \multicolumn{2}{|l|}{ Tumor size $(\mathrm{mm} ; \mathrm{n}=76)$} \\
\hline Median (range) & $30(1-135)$ \\
\hline$\leq 5$ & $71(93.4)$ \\
\hline$>5$ & $5(6.6)$ \\
\hline \multicolumn{2}{|l|}{ SEER stage $(n=98)$} \\
\hline Localized & $68(69.4)$ \\
\hline Regional & $23(23.5)$ \\
\hline Distant & $7(7.1)$ \\
\hline \multicolumn{2}{|l|}{ Tumor stage $(n=45)$} \\
\hline TI & $0(0)$ \\
\hline $\mathrm{T} 2$ & $35(77.8)$ \\
\hline T3 & $7(15.6)$ \\
\hline $\mathrm{T} 4$ & $3(6.7)$ \\
\hline \multicolumn{2}{|l|}{ Nodal stage $(n=47)$} \\
\hline Negative & $47(100)$ \\
\hline Positive & $0(0)$ \\
\hline \multicolumn{2}{|l|}{ Surgery $(n=100)$} \\
\hline No & $44(44)$ \\
\hline Yes & $56(56)$ \\
\hline \multicolumn{2}{|l|}{ Radiotherapy (n=99) } \\
\hline No & $13(13.1)$ \\
\hline Yes & $86(86.9)$ \\
\hline \multicolumn{2}{|l|}{ Local treatment strategy $(n=92)$} \\
\hline Primary surgery & $6(6.5)$ \\
\hline Primary RT & $36(39.1)$ \\
\hline Surgery + RT & $50(54.3)$ \\
\hline \multicolumn{2}{|l|}{ Cause of death $(n=9)$} \\
\hline Disease of heart & I (II.I) \\
\hline Disease of miscellaneous malignant cancer & $3(33.3)$ \\
\hline Disease of soft tissue including heart malignant cancer & $2(22.2)$ \\
\hline Diseases of kidney and renal pelvis malignant cancer & I (II.I) \\
\hline $\begin{array}{l}\text { Diseases of brain and other nervous system malignant } \\
\text { cancers }\end{array}$ & I (II.I) \\
\hline Diseases of eye and orbit malignant tumor-related diseases & I (II.I) \\
\hline
\end{tabular}

Abbreviations: RT, radiotherapy; SEER, Surveillance Epidemiology and End Results. and OS (Table 3). Furthermore, the local treatment strategy did not significantly affect CSS (log-rank test, $P=0.29$ ) or OS (log-rank test, $P=0.468$; Figure $2 \mathrm{~A}$ and $\mathrm{B}$ ).

\section{Discussion}

In this study, we used a population-based approach to investigate the clinicopathologic characteristics and prognoses of patients with orbital embryonal rhabdomyosarcoma and assess their survival outcomes according to different local treatment strategies. We observed that the disease occurred much more frequently in younger children and was mostly poorly differentiated/undifferentiated. In addition, we found that the local treatment strategy had no effect on survival outcomes. However, the condition is associated with an excellent prognosis.

The median age of patients diagnosed with orbital rhabdomyosarcoma is reported to range from 4 to 8 years, and its prevalence is higher in boys than in girls., ${ }^{2,13-15}$ Van Rijn et $\mathrm{a} \mathrm{l}^{16}$ found that the incidence of orbital rhabdomyosarcoma is higher in Caucasians, and that $70 \%$ of cases arise in nonHispanic white individuals. In accordance with this study, our study also confirmed the increased prevalence of the disease in boys and white patients. In addition, most of the patients included in our study exhibited poorly differentiated/ undifferentiated malignant tumors, in line with the highly malignant and aggressive characteristics of orbital embryonal rhabdomyosarcoma. However, the patients with orbital embryonal rhabdomyosarcoma included in this study had an excellent prognosis, consistent with previous studies. . $3,3,13,17-21^{-1}$ The improved OS of patients with orbital embryonal rhabdomyosarcoma can be attributed to the rapid development of comprehensive, multidisciplinary treatment.

The treatment protocols for orbital rhabdomyosarcoma were designed according to the clinical grouping system of the IRS and TNM classification. In the IRS clinical grouping system, orbital rhabdomyosarcoma is divided into 4 stages, IRS-I, IRS-II, IRS-III, and IRS-IV, and the recommended treatment of orbital rhabdomyosarcoma is based on the results of IRS-V study. ${ }^{8}$ Intensive treatment of patients at stages IRS-III and IRS-IV significantly improves their survival outcomes. Currently, the comprehensive treatment strategy for orbital embryonal rhabdomyosarcoma includes local surgery, RT, chemotherapy, and biotherapy. The surgical treatment of orbital rhabdomyosarcoma comprises tumorectomy, biopsy, and needle-aspiration biopsy. Prior to the 1960s, the standard treatment for orbital embryonal rhabdomyosarcoma was whole orbital exenteration, but the survival outcomes were unsatisfactory. ${ }^{22}$ 
Table 2 Patient characteristics according to local treatment strategies

\begin{tabular}{|c|c|c|c|c|}
\hline Characteristics & Surgery (\%) & RT (\%) & Surgery + RT (\%) & $P$-value \\
\hline \multicolumn{5}{|l|}{ Years of study $(n=92)$} \\
\hline $1988-1992$ & I (I6.7) & $2(5.6)$ & $7(14)$ & 0.389 \\
\hline $1993-1997$ & I (I6.7) & $4(I I . I)$ & $9(18)$ & \\
\hline $1998-2002$ & $2(33.3)$ & $10(27.8)$ & $5(10)$ & \\
\hline $2003-2007$ & I (16.7) & $10(27.8)$ & $13(26)$ & \\
\hline $2008-2012$ & I (16.7) & $10(27.8)$ & $16(32)$ & \\
\hline \multicolumn{5}{|l|}{ Age (years; $n=92$ ) } \\
\hline$\leq 6$ & $2(33.3)$ & $19(52.8)$ & $21(42)$ & 0.536 \\
\hline$>6$ & $4(66.7)$ & $17(47.2)$ & $29(58)$ & \\
\hline \multicolumn{5}{|l|}{ Race $(n=92)$} \\
\hline White & $4(66.7)$ & $30(83.3)$ & $40(80)$ & 0.343 \\
\hline Black & $2(33.3)$ & $2(5.6)$ & $6(12)$ & \\
\hline Others & $0(0)$ & $4(11.1)$ & $4(8)$ & \\
\hline \multicolumn{5}{|l|}{$\operatorname{Sex}(n=92)$} \\
\hline Male & $3(50)$ & $17(47.2)$ & $33(66)$ & 0.198 \\
\hline Female & $3(50)$ & $19(52.8)$ & $17(34)$ & \\
\hline \multicolumn{5}{|l|}{ Grade $(n=19)$} \\
\hline Well-differentiated & $0(0)$ & $0(0)$ & $0(0)$ & 1.000 \\
\hline Moderately differentiated & $0(0)$ & I (I6.7) & I (7.7) & \\
\hline Poorly differentiated & $0(0)$ & $3(50)$ & $8(6 \mid .5)$ & \\
\hline Undifferentiated & $0(0)$ & $2(33.3)$ & $4(30.8)$ & \\
\hline \multicolumn{5}{|l|}{ Tumor diameter $(\mathrm{cm} ; \mathrm{n}=7 \mathrm{l})$} \\
\hline$\leq 5$ & $5(100)$ & $23(88.5)$ & $40(100)$ & 0.086 \\
\hline$>5$ & $0(0)$ & $3(11.5)$ & $0(0)$ & \\
\hline \multicolumn{5}{|l|}{ SEER stage $(n=90)$} \\
\hline Localized & $3(50)$ & $25(69.4)$ & $35(72.9)$ & 0.644 \\
\hline Regional & $3(50)$ & $9(25)$ & $10(20.8)$ & \\
\hline Distant & $0(0)$ & $2(5.6)$ & $3(6.3)$ & \\
\hline \multicolumn{5}{|l|}{ Tumor stage $(n=43)$} \\
\hline TI & $0(0)$ & $0(0)$ & $0(0)$ & 0.400 \\
\hline $\mathrm{T} 2$ & I (50) & $13(86.7)$ & $19(73.1)$ & \\
\hline T3 & I (50) & $2(13.3)$ & $4(15.4)$ & \\
\hline $\mathrm{T} 4$ & $0(0)$ & $0(0)$ & $3(11.5)$ & \\
\hline \multicolumn{5}{|l|}{ Nodal stage $(n=45)$} \\
\hline Negative & $2(100)$ & $17(100)$ & $26(100)$ & - \\
\hline Positive & $0(0)$ & $0(0)$ & $0(0)$ & \\
\hline
\end{tabular}

Abbreviations: RT, radiotherapy; SEER, Surveillance Epidemiology and End Results.

In recent studies, approximately $20 \%$ of newly diagnosed patients and $50 \%$ of patients with recurrence received orbital exenteration. ${ }^{13,15,23} \mathrm{RT}$ is an important part of multidisciplinary therapy in patients with a poor prognosis after primary surgery. ${ }^{24-26}$ Cassady et a ${ }^{27}$ reported that high-dose RT decreases disease recurrence after orbital exenteration. In addition, Olivier Pascual et $\mathrm{al}^{7}$ found that early RT and complete tumorectomy may be important for the treatment of orbital rhabdomyosarcoma. However, patients who undergo primary surgery suffer blindness, disfigurement, and pain, which significantly affects their quality of life. In recent decades, the treatment of orbital embryonal rhabdomyosarcoma has adopted a more conservative approach, combining systemic chemotherapy and RT. ${ }^{28,29}$
In this study, most patients received chemotherapy and $54.3 \%$ of patients underwent surgery plus RT; however, the local treatment strategy, including primary surgery, primary RT, and surgery plus RT, had no effect on survival outcomes. Boutroux et $\mathrm{al}^{21}$ examined 95 patients with orbital rhabdomyosarcoma, including those with embryonal subtype, and found that RT as part of the first-line treatment was a significantly favorable prognostic factor for 5-year event-free survival (EFS), but not for OS. An international collaboration by 4 groups examined 306 patients who received multiagent chemotherapy, $80 \%$ of whom also underwent RT: their results showed that local recurrence in the RT group was lower than in the non-RT group ( $8 \%$ vs $44 \%$ ), whereas the EFSs of RT and non-RT patients were $82 \%$ and $53 \%$, respectively 
A

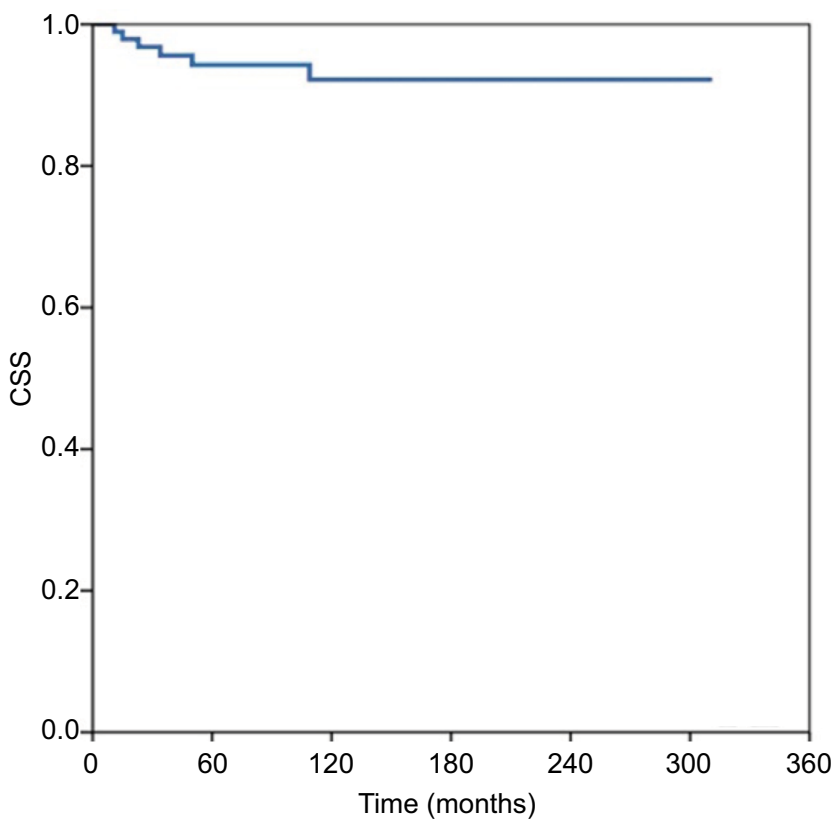

B

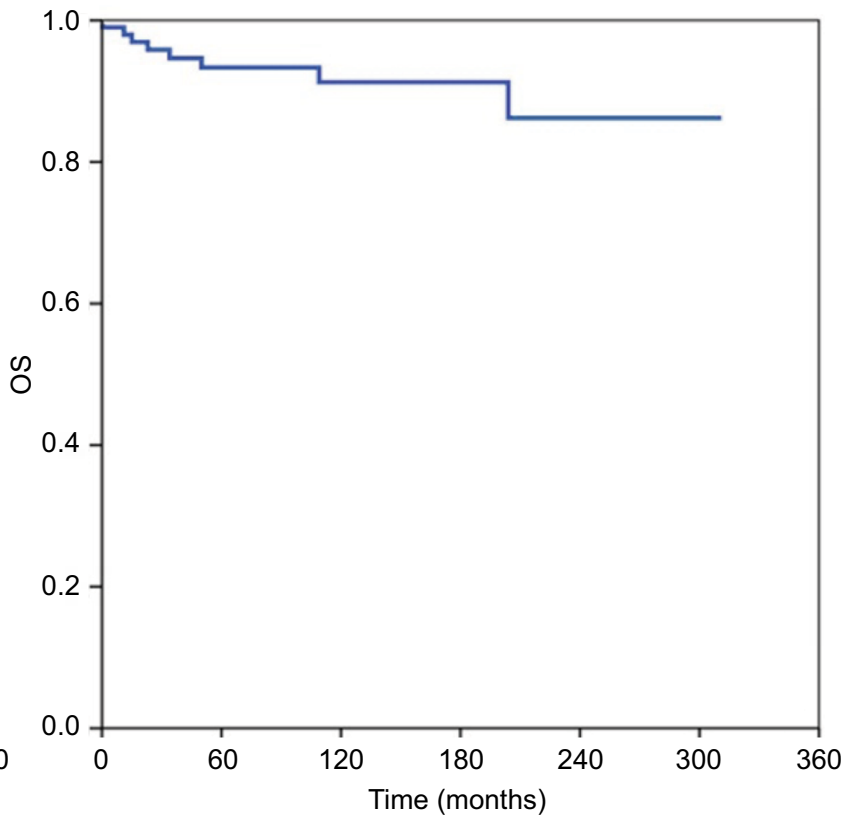

Figure I The CSS (A) and OS (B) of 102 patients with orbital embryonal rhabdomyosarcoma.

Abbreviations: CSS, cause-specific survival; OS, overall survival.

Table 3 Univariate analysis of the CSS and OS of 95 patients who were followed up for at least 12 months

\begin{tabular}{|c|c|c|c|c|c|c|}
\hline \multirow[t]{2}{*}{ Characteristics } & \multicolumn{3}{|l|}{ CSS } & \multicolumn{3}{|l|}{ OS } \\
\hline & HR & $95 \% \mathrm{Cl}$ & $P$-value & HR & $95 \% \mathrm{Cl}$ & $P$-value \\
\hline \multicolumn{7}{|l|}{ Age (years) } \\
\hline$\leq 6$ & 1 & & & I & & \\
\hline$>6$ & 0.561 & $0.094-3.36$ & 0.527 & 0.417 & $0.076-2.279$ & 0.313 \\
\hline \multicolumn{7}{|l|}{ Race } \\
\hline White & I & & & 1 & & \\
\hline Black & 3.543 & $0.592-21.226$ & 0.166 & 2.806 & $0.512-15.373$ & 0.235 \\
\hline Others & - & & 0.991 & - & - & 0.990 \\
\hline \multicolumn{7}{|l|}{ Sex } \\
\hline Male & I & & & I & & \\
\hline Female & 0.350 & $0.039-3.139$ & 0.348 & 0.285 & $0.033-2.446$ & 0.252 \\
\hline \multicolumn{7}{|c|}{ Tumor diameter $(\mathrm{cm})$} \\
\hline$\leq 5$ & $\mathrm{I}$ & & & 1 & & \\
\hline$>5$ & 0.044 & - & 0.797 & 0.044 & - & 0.797 \\
\hline \multicolumn{7}{|l|}{ SEER stage } \\
\hline Localized & 1 & & & I & & \\
\hline Regional & 5.370 & $0.487-59.276$ & 0.170 & 2.324 & $0.322-16.76 \mid$ & 0.403 \\
\hline Distant & - & - & 0.995 & - & - & 0.992 \\
\hline \multicolumn{7}{|c|}{ Local treatment strategy } \\
\hline Primary surgery & $\mathrm{I}$ & & & I & & \\
\hline Primary RT & 0.349 & $0.032-3.849$ & 0.390 & 0.385 & $0.035-4.282$ & 0.438 \\
\hline Surgery + RT & 0.135 & $0.008-2.16$ & 0.157 & $0.24 I$ & $0.022-2.675$ & 0.246 \\
\hline
\end{tabular}

Note: “-” indicates no data.

Abbreviations: $\mathrm{Cl}$, confidence interval; CSS, cause-specific survival; HR, hazard ratio; OS, overall survival; RT, radiotherapy; SEER, Surveillance Epidemiology and End Results.

$(P<0.001)$. However, there was no significant difference in OS between the RT and non-RT patients ( $87 \%$ vs $86 \%) .{ }^{30}$ Therefore, RT has a positive effect on EFS, but not on OS, which may be explained by the different effects of systemic and local treatment after disease recurrence. The choice of optimal local treatment strategies for orbital embryonal rhabdomyosarcoma should consider not only the local control, but also the potential damage to patients. However, the local treatment of orbital embryonal rhabdomyosarcoma remains an issue of controversy between European and North American 
A

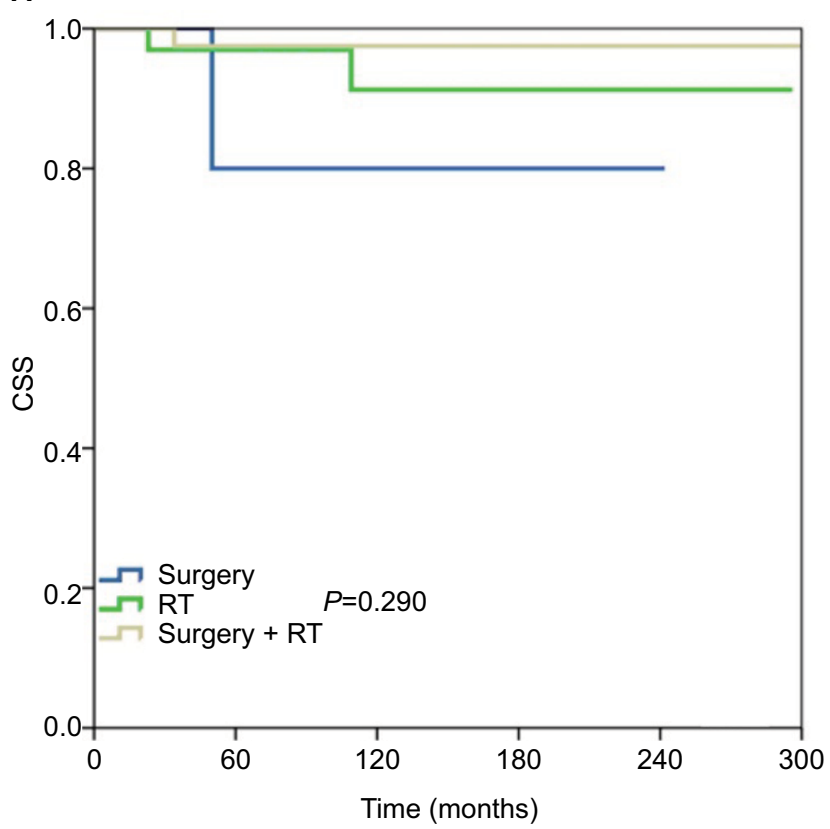

B

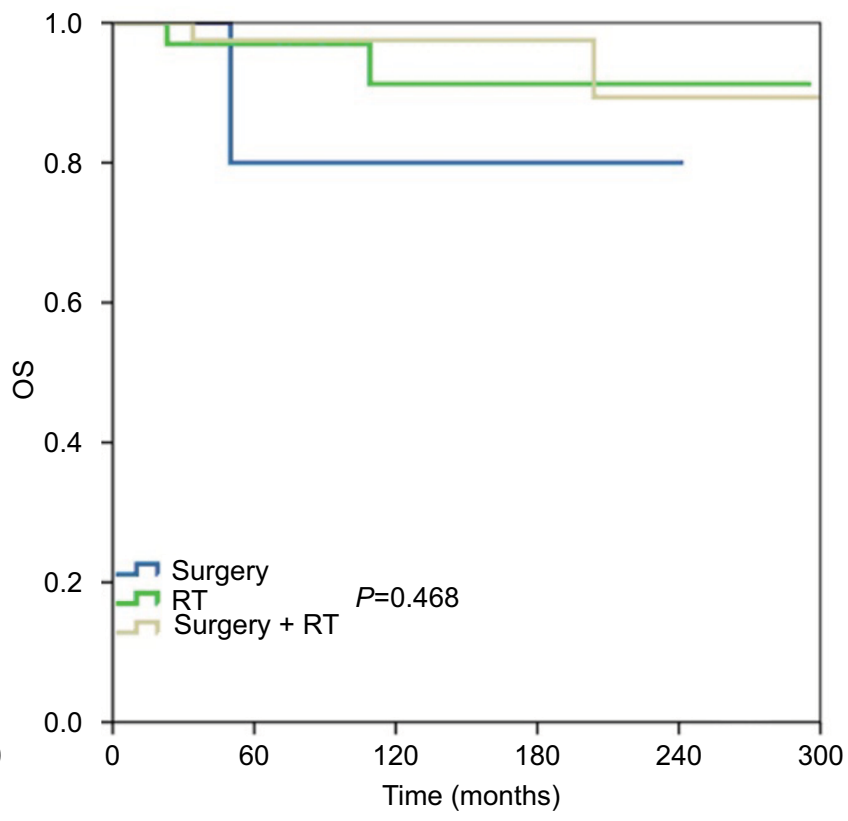

Figure 2 The CSS (A) and OS (B) of 95 patients with orbital embryonal rhabdomyosarcoma who were treated using different local treatment strategies and followed up for at least 12 months.

Abbreviations: CSS, cause-specific survival; OS, overall survival; RT, radiotherapy.

clinicians: the European approach attempts to avoid radiationinduced sequelae, whereas the North American approach aims to reduce disease recurrence. ${ }^{12}$

Rhabdomyosarcomas are sensitive to RT. Therefore, RT plays an important role in the local treatment of this disease. ${ }^{31}$ However, short- and long-term adverse effects commonly occur after RT, such as cataracts, xerophthalmia, chronic keratitis, orbital hypoplasia, corneal ulcers, vitreous hemorrhage, hypopituitarism, retinopathy, and uveitis..$^{24,27,32}$ Great progress in RT techniques has been made in recent years. Intensity-modulated RT is superior to conventional RT because of its higher treatment accuracy and better protection of normal organs. ${ }^{33}$ In addition, proton therapy may further lower the integral dose and spare normal tissues compared with intensity-modulated RT for orbital embryonal rhabdomyosarcoma. ${ }^{34}$ Interstitial brachytherapy is also an effective treatment for children with primary orbital rhabdomyosarcomas. ${ }^{35,36}$ Therefore, if treatment-related toxicity can be reduced through improvements in RT techniques, the North American approach may emerge as ethically superior, making RT a reasonable alternative to surgical treatment for orbital embryonal rhabdomyosarcoma.

\section{Limitations}

First, the retrospective nature of data from the SEER database and small sample size are major limitations. Second, detailed data related to the IRS clinical grouping system and the chemotherapy and RT regimens used are lacking in the SEER database, and most patients lack data on TNM classification. Therefore, we were unable to include these data and conduct further analyses. In addition, the overall sensitivity of the RT data in the current SEER database is $80 \%$. However, the RT data have a high specificity. ${ }^{37}$ Third, we were unable to obtain data on patterns of disease recurrence, complications after RT, and treatment results after recurrence.

\section{Conclusion}

Orbital embryonal rhabdomyosarcoma is a rare childhood malignant tumor with an excellent prognosis. There is no local treatment of choice for orbital embryonal rhabdomyosarcoma in terms of survival. However, following rapid progress in RT techniques, RT is a reasonable alternative therapy to surgical treatment. Further studies are needed to confirm our results.

\section{Disclosure}

The authors report no conflicts of interest in this work.

\section{References}

1. Exelby PR. Management of embryonal rhabdomyosarcoma in children. Surg Clin North Am. 1974;54:849-857.

2. Shields JA, Shields CL. Rhabdomyosarcoma: review for the ophthalmologist. Surv Ophthalmol. 2003;48(1):39-57.

3. Karcioglu ZA, Hadjistilianou D, Rozans M, DeFrancesco S. Orbital rhabdomyosarcoma. Cancer Control. 2004;11(5):328-333. 
4. Conneely MF, Mafee MF. Orbital rhabdomyosarcoma and simulating lesions. Neuroimaging Clin NAm. 2005;15(1):121-136.

5. Breneman JC, Lyden E, Pappo AS, et al. Prognostic factors and clinical outcomes in children and adolescents with metastatic rhabdomyosarcoma - a report from the Intergroup Rhabdomyosarcoma Study IV. $J$ Clin Oncol. 2003;21(1):78-84.

6. Neudorfer M, Leibovitch I, Stolovitch C, et al. Intraorbital and periorbital tumors in children - value of ultrasound and color Doppler imaging in the differential diagnosis. Am JOphthalmol. 2004;137(6):1065-1072.

7. Olivier Pascual N, Calvo JM, Abelairas Gómez JM. Rabdomiosarcoma orbitario: dificultades en el Protocolo de Tratamiento Europeo [Orbital rhabdomyosarcoma: difficulties with European treatment protocol]. Arch Soc Esp Oftalmol. 2005;80(6):331-338. Spanish.

8. Raney RB, Maurer HM, Anderson JR, et al. The Intergroup Rhabdomyosarcoma Study Group (IRSG): major lessons from the IRS-I through IRS-IV studies as background for the current IRS-V treatment protocols. Sarcoma. 2001;5(1):9-15.

9. Wharam MD, Hanfelt JJ, Tefft MC, et al. Radiation therapy for rhabdomyosarcoma: local failure risk for clinical group III patients on Intergroup Rhabdomyosarcoma Study II. Int J Radiat Oncol Biol Phys. 1997;38(4):797-804.

10. Shields CL, Shields JA, Honavar SG, et al. Primary ophthalmic rhabdomyosarcoma in 33 patients. Trans Am Ophthalmol Soc. 2001;99:133142; discussion 142-143.

11. Farnia B, Majumder MA, Paulino AC. Ethical analysis as a tool for addressing treatment controversies: radiotherapy timing in children with orbital rhabdomyosarcoma as a case example. $\mathrm{J} \mathrm{Am} \mathrm{Coll} \mathrm{Radiol.}$ 2015;12(5):484-490.

12. National Cancer Institute; Surveillance, Epidemiology, and End Results (SEER) Program [homepage on the Internet]. SEER*Stat Database: Incidence - SEER 18 Regs Custom Data (with chemotherapy recode), November 2015 Sub (2000-2013) <Katrina/Rita Population Adjustment> - Linked To County Attributes - Total U.S, 1969-2014 Counties, National Cancer Institute, DCCPS, Surveillance Research Program, released July 2016, based on the November 2015 submission. Available from: https://www.seer.cancer.gov. Accessed March 04, 2017.

13. Eade E, Tumuluri K, Do H, Rowe N, Smith J. Visual outcomes and late complications in paediatric orbital rhabdomyosarcoma. Clin Exp Ophthalmol. 2017;45(2):168-173

14. Ge X, Huang DS, Shi JT, et al. Multidisciplinary collaborative therapy for 30 children with orbital rhabdomyosarcoma. Asian Pac J Cancer Prev. 2013;14(8):4641-4646.

15. Raney B, Huh W, Hawkins D, et al. Outcome of patients with localized orbital sarcoma who relapsed following treatment on Intergroup Rhabdomyosarcoma Study Group (IRSG) Protocols-III and -IV, 1984-1997: a report from the Children's Oncology Group. Pediatr Blood Cancer. 2013;60(3):371-376

16. Van Rijn RR, Wilde JC, Bras J, Oldenburger F, McHugh KM, Merks $\mathrm{JH}$. Imaging findings in noncraniofacial childhood rhabdomyosarcoma. Pediatr Radiol. 2008;38(6):617-634.

17. George JL, Marchal JC. Les tumeurs d'orbite de l'enfant : examen clinique, paraclinique, diagnostic et particularités évolutives [Orbital tumors in children: clinical examination, imaging, specific progression] Neurochirurgie. 2010;56(2-3):244-248. French.

18. Forstner D, Borg M, Saxon B. Orbital rhabdomyosarcoma: multidisciplinary treatment experience. Australas Radiol. 2006;50(1):41-45.

19. Silvana G, Roberto de B, Domenico P, Giuseppina S, Ciracì L, Antonio C. Orbital cavitary rhabdomyosarcoma: a diagnostic dilemma. Orbit. 2010;29(1):45-47.
20. Hosoi H, Teramukai S, Matsumoto Y, et al. A review of 331 rhabdomyosarcoma cases in patients treated between 1991 and 2002 in Japan. Int J Clin Oncol. 2007;12(2):137-145.

21. Boutroux H, Cellier C, Mosseri V, et al. Orbital rhabdomyosarcoma in children: a favorable primary suitable for a less-invasive treatment strategy. J Pediatr Hematol Oncol. 2014;36(8):605-612.

22. Jones IS, Reese AB, Kraut J. Orbital rhabdomyosarcoma. An analysis of 62 cases. Am J Ophthalmol. 1966;61(4):721-736.

23. Bravo-Ljubetic L, Peralta-Calvo J, Larrañaga-Fragoso P, Pascual NO, Pastora-Salvador N, Gomez JA. Clinical management of orbital rhabdomyosarcoma in a referral center in Spain. J Pediatr Ophthalmol Strabismus. 2016;53(2):119-126.

24. Sagerman RH, Cassady JR, Tretter P. Radiation therapy for rhabdomyosarcoma of the orbit. Trans Am Acad Ophthalmol Otolaryngol. 1968;72(6):849-854.

25. Abramson DH, Ellsworth RM, Tretter P, Wolff JA, Kitchin FD. The treatment of orbital rhabdomyosarcoma with irradiation and chemotherapy. Ophthalmology. 1979;86(7):1330-1335.

26. Jereb B, Haik BG, Ong R, Ghavimi F. Parameningeal rhabdomyosarcoma (including the orbit): results of orbital irradiation. Int J Radiat Oncol Biol Phys. 1985;11(12):2057-2065.

27. Cassady JR, Sagerman RH, Tretter P, et al. Radiation therapy for rhabdomyosarcoma. Radiology. 1968;91(1):116-120.

28. Walterhouse DO, Pappo AS, Meza JL, et al. Shorter-duration therapy using vincristine, dactinomycin, and lower-dose cyclophosphamide with or without radiotherapy for patients with newly diagnosed low-risk rhabdomyosarcoma: a report from the Soft Tissue Sarcoma Committee of the Children's Oncology Group. J Clin Oncol. 2014;32(31):3547-3552.

29. Raney RB, Walterhouse DO, Meza JL, et al. Results of the Intergroup Rhabdomyosarcoma Study Group D9602 protocol, using vincristine and dactinomycin with or without cyclophosphamide and radiation therapy, for newly diagnosed patients with low-risk embryonal rhabdomyosarcoma: a report from the Soft Tissue Sarcoma Committee of the Children's Oncology Group. JClin Oncol. 2011;29(10):1312-1318.

30. Oberlin O, Rey A, Anderson J, et al. Treatment of orbital rhabdomyosarcoma: survival and late effects of treatment - results of an international workshop. J Clin Oncol. 2001;19(1):197-204.

31. Goto T, Kosaku H, Kobayashi H, Hozumi T, Kondo T. [Soft tissue sarcoma: postoperative chemotherapy]. Gan To Kagaku Ryoho. 2004;31(9):1324-1330. Japanese.

32. Raney RB, Anderson JR, Kollath J, et al. Late effects of therapy in 94 patients with localized rhabdomyosarcoma of the orbit: report from the Intergroup Rhabdomyosarcoma Study (IRS)-III, 1984-1991. Med Pediatr Oncol. 2000;34(6):413-420.

33. Wolden SL, La TH, LaQuaglia MP, Meyers PA, Kraus DH, Wexler LH Long-term results of three-dimensional conformal radiation therapy for patients with rhabdomyosarcoma. Cancer. 2003;97(1):179-185.

34. Ladra MM, Edgington SK, Mahajan A, et al. A dosimetric comparison of proton and intensity modulated radiation therapy in pediatric rhabdomyosarcoma patients enrolled on a prospective phase II proton study. Radiother Oncol. 2014;113(1):77-83.

35. Ge X, Ma J, Dai H, Ren L, Li Q, Shi J. Clinical research on the treatment effects of radioactive (125)I seeds interstitial brachytherapy on children with primary orbital rhabdomyosarcoma. Med Oncol. 2014;31(9):27.

36. Schoot RA, Saeed P, Freling NJ, et al. Local resection and brachytherapy for primary orbital rhabdomyosarcoma: outcome and failure pattern analysis. Ophthalmic Plast Reconstr Surg. 2016;32(5):354-360.

37. Noone AM, Lund JL, Mariotto A, et al. Comparison of SEER treatment data with medicare claims. Med Care. 2016;54(9):e55-e64. 


\section{Publish your work in this journal}

Cancer Management and Research is an international, peer-reviewed open access journal focusing on cancer research and the optimal use of preventative and integrated treatment interventions to achieve improved outcomes, enhanced survival and quality of life for the cancer patient. The manuscript management system is completely online and includes a very quick and fair peer-review system, which is all easy to use. Visit http://www.dovepress.com/testimonials.php to read real quotes from published authors. 